\title{
Are We Ready for Unmanned Surface Vehicles in Inland Waterways? The USVInland Multisensor Dataset and Benchmark
}

\author{
Yuwei Cheng ${ }^{1}$, Mengxin Jiang ${ }^{2}$, Jiannan $\mathrm{Zhu}^{3}$, Yimin $\mathrm{Liu}^{4}$
}

\begin{abstract}
Unmanned surface vehicles (USVs) have great value with their ability to execute hazardous and timeconsuming missions over water surfaces. Recently, USVs for inland waterways have attracted increasing attention for their potential application in autonomous monitoring, transportation, and cleaning. However, unlike sailing in open water, the challenges posed by scenes of inland waterways, such as the complex distribution of obstacles, the global positioning system (GPS) signal denial environment, the reflection of bank-side structures, and the fog over the water surface, all impede USV application in inland waterways. To address these problems and stimulate relevant research, we introduce USVInland, a multisensor dataset for USVs in inland waterways. The collection of USVInland spans a trajectory of more than 26 $\mathrm{km}$ in diverse real-world scenes of inland waterways using various modalities, including lidar, stereo cameras, millimeterwave radar, GPS, and inertial measurement units (IMUs). Based on the requirements and challenges in the perception and navigation of USVs for inland waterways, we build benchmarks for simultaneous localization and mapping (SLAM), stereo matching, and water segmentation. We evaluate common algorithms for the above tasks to determine the influence of unique inland waterway scenes on algorithm performance. Our dataset and the development tools are available online at https://www.orca-tech.cn/datasets.html.
\end{abstract}

Index Terms-Datasets for SLAM, datasets for robotic vision, marine robotics

\section{INTRODUCTION}

In recent years, unmanned surface vehicles (USVs), also known as autonomous surface vehicles (ASVs), have gained increasing prominence driven by their ability to perform hazardous and time-consuming missions [1]. The strong demands from commercial, scientific and environmental communities accelerate the development of USV applications, such as hydrographic surveying and charting, marine resource explorations, water quality monitoring, and floating waste removal [2]-[5]. Compared to coastal and marine USVs, USVs for inland waterways are more closely related to human life and have a large potential value, such as

\footnotetext{
${ }^{1}$ Yuwei Cheng is with the Department of Electronic Engineering, Tsinghua University, Beijing, 100084 China, and also with the ORCA-TECH, Shaanxi, 710075 China chengyw18@mails.tsinghua.edu.cn

${ }^{2}$ Mengxin Jiang is with the Department of Automation, Tsinghua University, Beijing, 10084 China, and also with the ORCA-TECH, Shaanxi, 710075 China jiangmx16@mails.tsinghua.edu.cn

${ }^{3}$ Jiannan Zhu is with the School of Marine Science and Technology, Northwestern Polytechnical University, Shaanxi, 710109 China, and also with the ORCA-TECH, Shaanxi, 710075 China jacknyzhu@orca-tech.com.cn

${ }^{4}$ Yimin Liu, as the corresponding author, is with the Department of Electronic Engineering, Tsinghua University, Beijing, 100084 China yiminliuetsinghua.edu.cn
}

being the core of building autonomous transportation systems in inland waterways [6]. Despite this, USVs for inland waterways have not been fully developed mainly due to the challenges of the variable and complex real-world driving scenes of inland waterways [6], as shown in Fig. 11 In narrow inland waterways, global positioning system (GPS) signals are sometimes attenuated due to the occlusion of riparian vegetation, bridges, and urban settlements [7]. In this case, to achieve reliable navigation in inland waterways, accurate and real-time localization relies on the estimation of the vehicle's relative location to the surrounding environment [8]. In addition, it is essential to keep the vehicle at a safe distance from the bank and other obstacles. Thus, similar to autonomous driving on roads, the tasks of simultaneous localization and mapping (SLAM), stereo matching, and water segmentation based on sensors such as lidar, stereo cameras, and millimeter-wave radar are introduced to support the localization, perception, and navigation of USVs for inland waterways.

However, in inland waterways, lidar data noise caused by fog over the water surface and strong light reflection would compromise the lidar system [9], [10]. In terms of the visual system, surface fog can reduce visibility and obscure scenes. The images might be overexposed due to sunlight reflection. In addition, the reflection of bankside objects and the rippling caused by raindrops also interfere with the visual system. Besides, in inland waterways, USVs pose changes more frequently due to the low surface friction and water waves, leading to difficulties in matching landmarks in adjacent frames for some SLAM algorithms. Common strategies for autonomous driving and marine USVs are infeasible for USVs in inland waterways.

Publicly available datasets can encourage breakthroughs and enable a fair comparison between different algorithms. For autonomous driving, advances have been achieved through exploration on some widespread datasets, such as the KITTI [11], RobotCar [12], and Cityscapes datasets [13]. Datasets such as the Stanford Drone Dataset [14] and the Okutama-Action dataset [15] also promote the development of unmanned aerial vehicles (UAVs). For USVs, datasets for certain tasks in marine and coastal areas, including object detection [16], [17] and water segmentation [18], [19], have been published. However, to the best of our knowledge, there is no public standard dataset aimed at USVs for inland waterways or a dataset for USVs containing data from various sensors that makes multiple tasks and sensor fusion methods possible.

To fill this gap and help research USVs, we introduce 


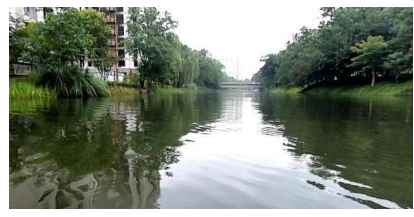

(a) Normal

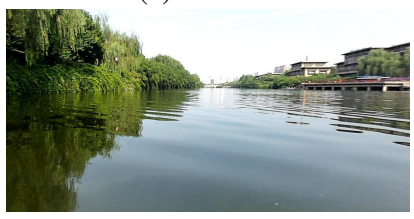

(c) Wide

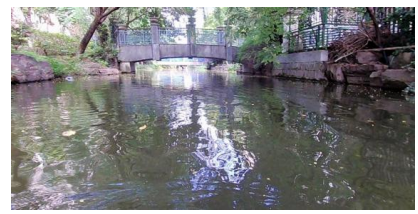

(b) Narrow

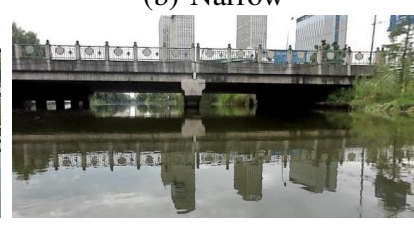

(d) Complex
Fig. 1: A variety of inland waterway landscapes and structures.

USVInland, a multisensor dataset of USVs for inland waterways collected under a variety of weather conditions. Typical sensors used in autonomous driving, including stereo cameras, a lidar system, GPS antennas, and inertial measurement units (IMUs), are mounted on our acquisition platform. Three millimeter-wave radars, which are less expensive and more robust to weather conditions than lidar systems, are also used to capture data of the environment around the vehicle. All the sensors are synchronized, and the calibration results are provided in our dataset.

Inspired by the KITTI visual benchmark [11], with reference to the tasks for the autonomous vehicle, we introduce SLAM, stereo matching, and water segmentation tasks into our dataset and build corresponding benchmarks. Experiments on different tasks are carried out on our dataset based on typical algorithms. The results show that the performance of commonly used algorithms would decrease due to the characteristics of inland waterway scenes.

The USVInland dataset along with the calibration data are available online on our website. To make the dataset more accessible to researchers, we provide development tools on the website for loading and visualizing synchronized data from multiple sensors. The introduction of the file formats can also be found on the website.

To summarize, this paper mainly contributes to the following aspects:

- We present the USVInland dataset, the first dataset for USVs in inland waterways, containing data from multiple sensors, including a lidar system, stereo cameras, millimeter-wave radar, GPS antennas, and IMUs.

- Our dataset is collected in real-world scenes of inland waterways at different times, covering a variety of waterway landscapes and weather conditions. We provide accurate timestamps for the synchronization of different sensors as well as intrinsic and extrinsic sensor calibration results.

- Aiming at the requirements and challenges in the perception and navigation of USVs for inland waterways, we introduce the tasks of SLAM, stereo matching, and water segmentation. We build corresponding benchmarks and evaluate common algorithms for different tasks on our benchmarks.

\section{RELATED WORK}

\section{A. USVs for the Inland Waterway}

With advances in autonomous vehicles, the potential applications of USVs in inland waterways have been proven to be achievable and partly converted into practical applications in fields of monitoring, cleaning, and transportation. Shojaei et al. [20] present their study on using USVs for structural health monitoring, which is based on detecting cracks in buildings near water bodies by visual information. The solarpowered Lake Wivenhoe USV was designed to monitor the water quality properties and greenhouse gas emissions [21]. The Water Environmental Mobile Observer (WeMo) developed by Madeo et al. [4] collects data on water depth in addition to water quality. For water body cleaning, the USVs presented in [22] and [5] were developed to collect floating waste above the water surface in inland waterways. In addition, the Roboat project [6], [10] aims at autonomous transportation and dynamic floating infrastructure construction in urban waterways. Their current boat includes a complete design of localization, perception, planning, and control to support autonomous transportation tasks.

\section{B. SLAM}

SLAM is an essential capability for the self-navigation of self-driving vehicles in an unknown environment. Based on lidar and cameras, constantly emerging approaches such as LOAM [23], Cartographer [24], and LeGO-LOAM [25] for lidar SLAM, as well as ORB-SLAM [26] and VINS [27] for visual SLAM, have been proven to be valuable for navigation systems of self-driving vehicles and have been transferred into practical use.

In recent years, $77 \mathrm{GHz}$ automotive radar technology has rapidly developed. The resolution of radar point clouds becomes higher, providing richer information on the surrounding environment. Meanwhile, some researchers pay attention to SLAM based on millimeter-wave radar [28]-[31]. It is worth investigating the application value of millimeter-wave radar on USVs because radar is more robust to harsh weather conditions, such as fog and rain than lidar systems and cameras and it is not influenced by lighting conditions.

For USVs, the SLAM algorithm has been used to overcome the difficulties of navigation in the GPS signal denial area. Han and Kim [32] proposed a method to estimate the location of the vehicle under bridges using a landmarkbased SLAM framework based on lidar point clouds. Wang et al. [10] applied LIO-SAM [33], a tightly-coupled Lidar inertial odometry, on their urban waterway transportation USV, Roboat II. Kriechbaumer et al. [7] focused on visual odometry used in USV localization, and they evaluate both the feature-based and appearance-based visual odometry algorithms to develop a river monitoring USV. Han et al. [1] proposed C-SLAM for USV navigation and mapping 
in coastal water, which is based on the coastline features extracted from a marine radar image.

\section{Stereo Matching}

Stereo matching, which is used to determine the disparity map between an image pair taken from the same scene for depth information [34], is always an essential task for the perception of self-driving vehicles. Classical methods such as Semi-Global Matching (SGM) [35] and recently proposed methods such as those from [36]-[38] enable increasingly accurate depth information of the surrounding environment for self-driving vehicles.

For USVs, stereo matching is widely used for obstacle detection. For example, Wang and Wei [39] developed a marine USV system for obstacle detection and tracking by building a grid map generated from stereo reconstructed 3D points. Based on the idea of combining semantic segmentation and stereo matching, the approach introduced in [40] performs well in segmenting obstacles in marine environments by aligning image pairs to enforce segmentation consistency.

\section{Water Segmentation}

Studies on detecting water area have been performed to enable safe navigation for both marine and inland waterways USVs. Most of the current detection methods are based on visual information. Zhan et al. [41] propose their method in which the waterline is fitted based on the extracted potential boundary points using random sample consensus (RANSAC). Another method described in [42] first extracts line segments in an image based on gradient value and then generates the onshore line segment pool to distinguish the shore area and water area.

However, the above methods may fail when the shoreline is extremely irregular in a complex inland water environment or when there is interference over the water surface. Recently, water area detection based on detecting the water boundaries has been treated as a water segmentation task based on deep learning. Zhan et al. [43] proposed an online learning approach to segment water, sky, and shore areas in unknown navigation environments using a convolutional neural network (CNN). Bovcon and Kristan [44] proposed a water-obstacle separation and refinement network that outperforms current semantic segmentation models. Based on the semantic segmentation model, water area detection is not limited to areas with straight boundaries.

\section{DATASET}

\section{A. Platform}

Our acquisition platform consists of an inflatable surface vehicle with multiple sensors that enables benchmarks for various tasks. The platform is equipped with a 16-beam lidar system, a stereo color camera, three millimeter-wave radars, and an inertial navigation system (INS). The locations of the sensors on our platform are shown in Fig. 2. A lidar system that captures accurate 3D properties around our boat is mounted on the top of the platform. The stereo camera is placed in front of the boat facing forward. As the horizontal

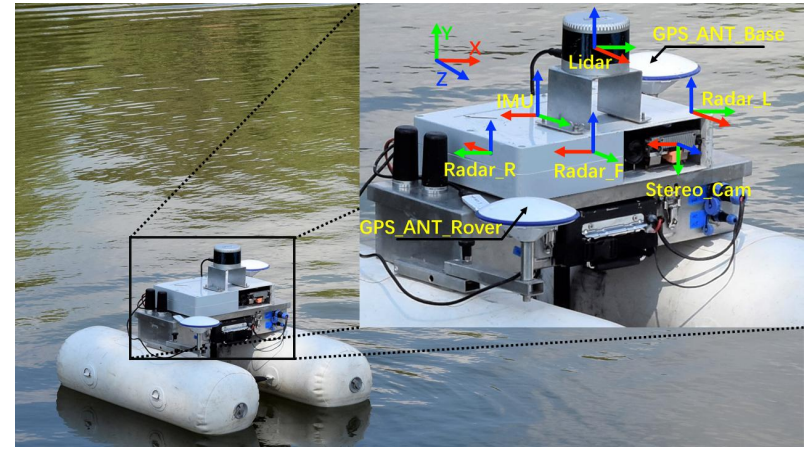

Fig. 2: The inflatable boat equipped with multiple sensors. The directions of the sensors are marked in different colors (X-red, Y-green, Z-blue).

field of view (HFoV) of the millimeter-wave radar is limited, we mounted three radars at the front and two sides of the acquisition setup to collect more comprehensive radar data around the vehicle. Two GPS antennas are mounted on two nonadjacent corners of the boat connected with the base and rover module inside of the GPS. One of them is set at the real-time kinematic (RTK) mode receiving signals from the base station to provide up to centimeter-level accuracy. The other module is connected with the rover module to obtain accurate absolute heading information by the moving base support in the module firmware [45].

Detailed information about the sensors of the platform is described as follows:

$1 \times$ Lidar: $905 \mathrm{~nm}, 16$ beams, HFoV $360^{\circ} \times$ Vertical Field of View (VFoV) $32^{\circ}, 10 \mathrm{~Hz}$, Max Range $100 \mathrm{~m}$, Range Resolution $2 \mathrm{~cm}$, Horizontal Angular Resolution $0.18^{\circ}$

$1 \times$ Stereo Camera with $1 \times$ IMU: RGB: $640 \times 400 / 1280$ $\times 800,20 \mathrm{~Hz}, \mathrm{HFoV} 95^{\circ} \times \mathrm{VFoV} 50^{\circ} \times$ Diagonal Field of View (DFoV) $112^{\circ}$, Global Shutter, $80 \mathrm{~mm}$ Baseline, Hardware Synchronized; IMU: $200 \mathrm{~Hz}$, 6-axis

$3 \times$ Radar: $10 \mathrm{~Hz}$, HFoV94 $4^{\circ} \times \mathrm{VFoV} 50^{\circ}(-6 \mathrm{~dB})$, Range Resolution 0.045m, Maximum Unambiguous Range 18.08m, Maximum Radial Velocity $3.96 \mathrm{~m} / \mathrm{s}$, Radial Velocity Resolution $0.25 \mathrm{~m} / \mathrm{s}$

$1 \times$ Inertial Navigation System: IMU $50 \mathrm{~Hz}, 6$-axis, $2 \times$ GPS: U-Blox ZED-F9, 5Hz, Receive RTK Signal, Include Moving Base Support

More information including the make and model of each sensor can be found on our website.

\section{B. Data Collection}

The USVInland dataset collection spans the period from May to August 2020. During this period, our inflatable boat was driven both manually and automatically along inland waterways in typical cities where there are rich hydrographic networks. Considering that harsh weather conditions dramatically increase the difficulties of autonomous driving especially in inland waterways, USVInland contains data collected under various weather conditions as shown in Fig. 3 , to cover real-world driving scenes. The waterways we 


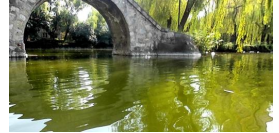

(a) Sun

(d) Mist

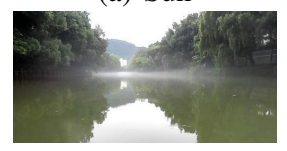

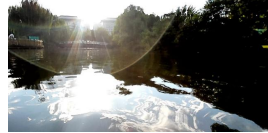

(b) Strong Light

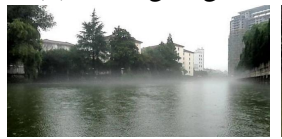

(e) Rain

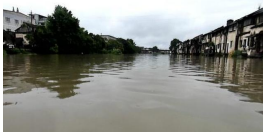

(c) Overcast

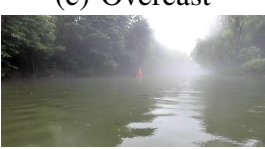

(f) Fog
Fig. 3: The dataset is collected under different weather conditions to reveal the challenges of USV perception and navigation in real-world inland waterways.

choose are of different widths and lengths with a variety of bankside landscapes. Part of the data collection route is shown in Fig. 4

\section{Synchronization and Calibration}

For synchronization, at the beginning of data collection, the absolute timestamps of the frames first captured by each sensor are recorded. Then, accurate relative timestamps of each frame from the clock inside each sensor are recorded together with the sensor data. By setting an absolute timestamp as the beginning and referring to the relative timestamps, the data from different sensors can be synchronized. For autonomous vehicle whose speed can be up to $30-55 \mathrm{~km} / \mathrm{h}$, a synchronization accuracy ranging from $0-7 \mathrm{~ms}$ can provide a dataset of high quality [46]. As the speed of our boat is much slower $(0-6.48 \mathrm{~km} / \mathrm{s})$, we believe that the synchronization accuracy of our dataset (a maximum synchronization drift of $25 \mathrm{~ms}$ between radar and camera) can meet the requirement of inland waterway scenes.

The intrinsic parameters of the stereo camera and the extrinsic parameters of cameras and the IMU inside the same device are from the factory calibration. As in the calibration process used in [28], coregistration process of the three sensors (lidar system, camera, radar) is split into two separate processes. First, we use a calibration tool [47] to calibrate the extrinsic parameters between the camera and lidar system. The approach is based on matching the automatically detected checkerboard corners in the image and the manually extracted lidar point clouds of the checkerboard. To register the radar with lidar, we measure the relative position of the three radars to lidar, which is then optimized by matching the lidar and radar point clouds of several physical targets. The raw dataset we used for calibration is also provided, if some researchers want to obtain calibration results on their own.

\section{Benchmark Selection}

The raw data we collect cover a trajectory of more than $26 \mathrm{~km}$ in total. There are 27 continuous raw sequences collected under different weather conditions. The width of the waterways varies from $10 \mathrm{~m}$ to $40 \mathrm{~m}$ with a length of the covered waterway ranging from $100 \mathrm{~m}$ to $3400 \mathrm{~m}$. For the three tasks, the data are extracted from the raw dataset.

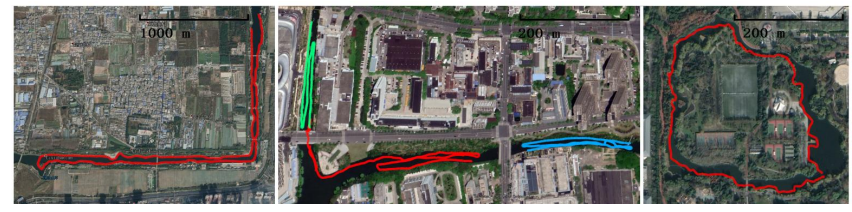

Fig. 4: Part of the collection trajectories ( 5 of the 27 raw sequences: $X 31 \_1$ in the image on the left, N02_1, N02_2 and N02_3 in the middle, $X 01 \_3$ in the image on the right) are shown here. Our dataset is collected in cities including Xi'an, Ningbo and Hangzhou in China. We name each sequence by the date and location of collection. More details about the data can be found on our website.

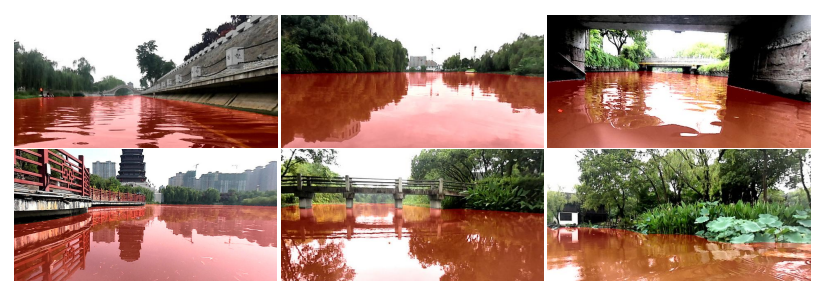

Fig. 5: The images shown in the figure represent typical waterline structures in our dataset: straight lines, nearly horizontal waterlines in particularly wide waterways, areas under bridges, irregular waterlines, multiple obstacles distributions, and full vegetation on banks. The water area is annotated in red.

Our SLAM benchmark consists of 33 public sequences in total, which are cut from the 27 raw sequences. The duration of each SLAM sequence ranges from 100 seconds to 2600 seconds. As the GPS signal might be unavailable when the boat is driving in some narrow waterways, the localization precision factor is included in the data during collection to avoid using low-precision location data as a reference. The GPS localization precision factor is recorded as RTK Fixed and RTK Float for different levels of accuracy. Only sequences with GPS data under RTK Fixed mode are selected for the SLAM benchmark.

Our stereo matching benchmark consists of 324 image pairs, which are selected by first downsampling the raw stereo images and applying the similar procedure described in [11] based on clustering for the sample diversity. Some extracted obscured and overexposed images are then excluded manually.

The water segmentation benchmark contains 700 images selected by manually selecting the images of waterways with various waterline shapes. In addition, the images we choose are captured under different weather or lighting conditions. Unlike most datasets used for marine water segmentation in which the waterline is mainly the straight water-sky line, our benchmark covers different landscapes of inland waterways with various waterline structures. Examples of our data are shown in Fig. 5 . 
TABLE I: Results on some of the sequences in our dataset.

\begin{tabular}{c|c|c|c|c|c}
\hline Sequence & Distance [m] & Loop Back & Weather & \multicolumn{2}{|c}{ Relative Pose Error* } \\
\cline { 5 - 6 } & & & & LOAM [23] & LeGO-LOAM [25] \\
\hline N02_6_1265_1431 & 143 & $\times$ & Sunny & $1.8 / 0.03$ & $1.7 / 0.09$ \\
H05_7_160_270 & 135 & $\times$ & Rain & $2.7 / 0.03$ & $2.8 / 0.08$ \\
N03_3_605_760 & 167 & $\times$ & Overcast & $1.2 / 0.04$ & $2.5 / 0.07$ \\
H05_8_30_205 & 229 & $\checkmark$ & Rain and Fog & $6.5 / 0.17$ & $4.8 / 0.18$ \\
N03_2_80_536 & 513 & $\checkmark$ & Overcast & $2.5 / 0.09$ & $2.6 / 0.10$ \\
H05_9_115_700 & 765 & $\checkmark$ & Mist & $2.6 / 0.02$ & $3.5 / 0.07$ \\
Average & $*$ & $*$ & $*$ & $2.9 / 0.06$ & $3.0 / 0.10$ \\
\hline
\end{tabular}

*The relative pose errors (RPEs) are measured using segments of trajectories at 5, 10, 50, 100, .., $400 \mathrm{~m}$ lengths: relative translational error in $\% /$ relative rotational error in degrees per $1 \mathrm{~m}$.
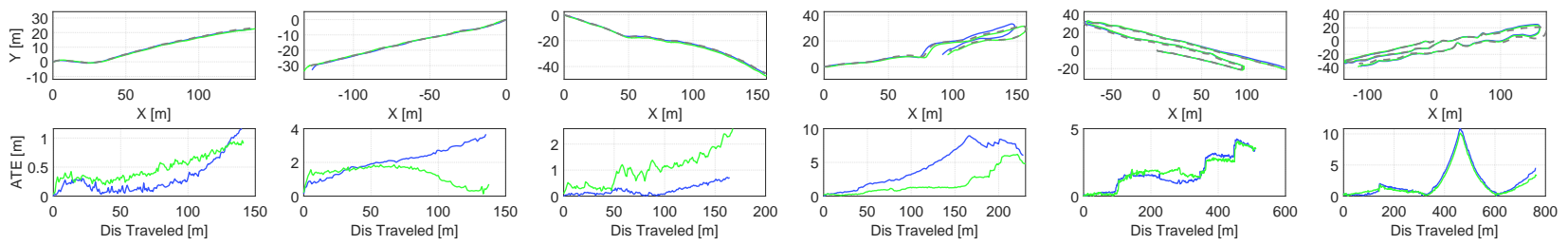

Fig. 6: The results of LOAM [23] and LeGO-LOAM [25] on 6 sequences (listed in in Table. If compared with the ground truth trajectories and the absolute trajectory errors (ATEs) as a function of distance traveled. The dashed lines correspond to the ground truth trajectories. The blue and green lines correspond to the results of LOAM and LeGO-LOAM respectively. (The trajectories are rotated to save space.)

\section{E. Ground-Truth Generation}

For SLAM, the ground-truth data come from the output of GPS location and IMU pose information.

To generate the ground truth for the stereo matching benchmark, synchronized lidar point cloud data that contain depth information are used. The lidar point clouds in a single frame are relatively sparse when projected onto the image. In this case, we integrate 3D lidar point clouds of adjacent frames based on the iterative closest point (ICP) [48] and then project the aggregated points onto the image plane for a denser ground truth. No depth ground truth of the water surface area is provided because there is a reduction in the intensity of the reflected light and nearly no lidar point cloud of the water surface is detected.

To generate ground truth for the water segmentation benchmark, the images are annotated manually by using the LabelMe toolbox [49]. The annotators are asked to label the water area by creating polygons covering the water segment. The annotation quality is ensured as all the annotations are repeatedly verified and corrected.

\section{EXPERIMENT}

Based on the three benchmarks, experiments are carried out to show that our dataset is valid and to evaluate the performance of relevant algorithms when applied to inland waterway scenes.

\section{A. SLAM}

For Lidar SLAM, we evaluated LOAM [23] and LeGOLOAM [25] on some of the sequences in our dataset. We selected 6 sequences collected under different weather conditions. The results are shown in Table. [1 and Fig. 6
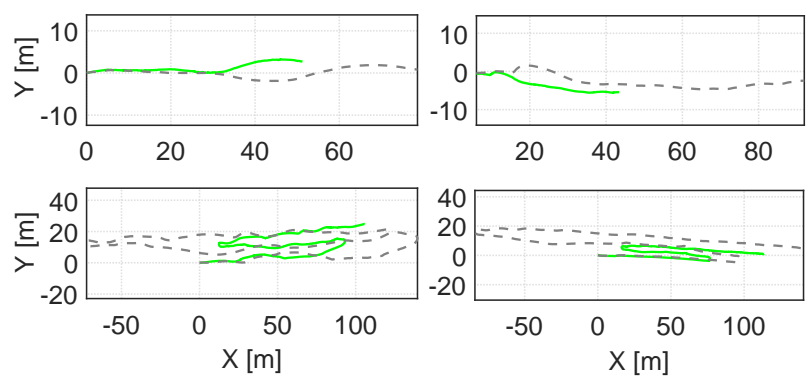

Fig. 7: Results of ORB-SLAM2 [50] on 4 sequences in our dataset: the dashed lines and the green lines correspond to the ground truth trajectories and the results of ORB-SLAM2 respectively. The results differ a lot from the ground truth trajectories. (The trajectories are rotated to save space. The 4 sequences are $W 06 \_2 \_57 \_115$, N03_4_440_523, H05_6_20_550, N03_2_80_536 respectively.)

As the boat sails over the flat and horizontal water surface, the evaluation is done in 2-D space. As can be seen, for sequences collected under harsh environments like rain and fog, there is a decrease in the performance of both LOAM and LeGO-LOAM.

The experiment for visual SLAM is based on ORBSLAM2 [50]. We test the algorithms on all the public 33 sequences in our dataset. The performance of the algorithm on our dataset is unacceptable and the estimated results differ a lot from the ground truth. The average relative translation error is more than 20\%. Part of the results and the ground truth trajectories are shown in Fig. 7 .

For visual SLAM, it can be observed from Fig. 8 that, 


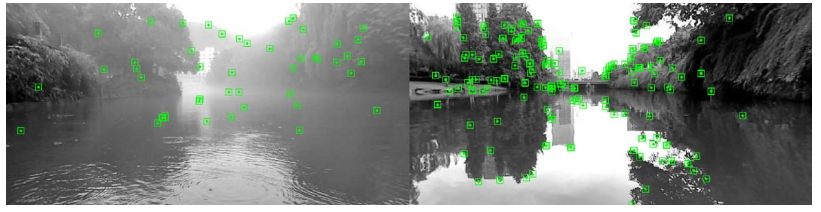

Fig. 8: Representative scenes for features detected in ORBSLAM2 [51]: the green points show the ORB features. The image on the left shows the detected feature points with fog over the surface. The image on the right shows that the reflection textures are detected as mapping features.
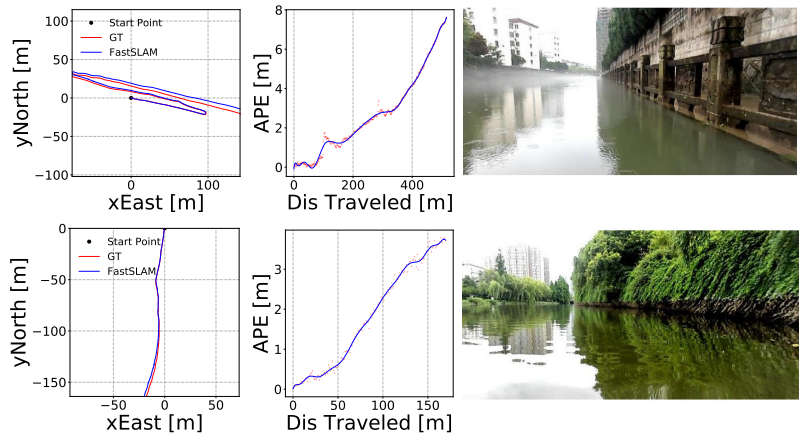

Fig. 9: Radar SLAM result: the figures on the left show the estimated results, the ground truth (GT) trajectories, and the absolute trajectory errors (ATEs) as a function of distance traveled. The images on the right show the corresponding scenes. The scene on top contains stone balustrades along the bank.After traveling the same distance, the ATE of the sequence on the top is much smaller than ATE of the one at the bottom. (The two sequences are H05_9_115_700 and H03_3_605_760 respectively. The red dots are ATEs and the curves are polynomial fitting results of the dots.)

on the one hand, the fog over the water surface can reduce visibility and lead to difficulties in detecting feature points. On the other hand, as mentioned in [52], the lack of salient features in short range (mainly extracted from river bank) reduces the performance of feature based algorithms. In our experiment, in inland waterways that are wide and when the vehicle is driving in the middle of the waterway, most of the extracted features are from the reflection on the water surface and are far away. The errors in depth estimation for the feature points at relatively far distances can lead to errors in relative pose estimation between adjacent frames. In addition, in some cases, the water wave can distort the water reflection, which makes the keypoints in the reflection unstable in adjacent frames. In this case, the estimated result differs greatly from the ground truth.

For radar SLAM, we evaluate the algorithm based on FastSLAM proposed by Schuster et al. [53] on our dataset. The radar data are the fusion of the point clouds from 3 millimeter-wave radars, which are mounted at the front and two sides of the platform for more comprehensive information around the vehicle. As the detection range of radar is limited, we select 8 sequences in which the boat sails close to the bankside or the rivers are narrow (the 8 sequences we selected are marked in our website). Testing on these sequences, we obtain a relative translational error of $3.95 \%$ (averaged over trajectories of 5 to $200 \mathrm{~m}$ length).

The results on two of the selected sequences are presented in Fig. 9 It can be observed that the algorithm achieves a better performance when applied to scenes with distinct targets on the waterway sides for radar, such as the stone balustrade, which contains metals. However, as shown in the first sequence of Fig. 9, when the boat turned around (at the turning point in the first sequence) and sailed in the middle of the waterway, being further to the waterway sides, the drift between the estimated result and ground truth grew a lot as the number of valid targets decreased.

\section{B. Stereo Matching}

For stereo matching, we apply SGM [35] on the 180 lowresolution $(640 \times 320)$ image pairs in our stereo matching benchmark. The SGM result is compared with the groundtruth disparity map generated by transforming the depth information from the lidar point clouds. Only pixels with ground-truth data are included in the evaluation. We calculate the error percentage as a function of the disparity error threshold. The result is shown in Table. II The SGM performance is not good when applied to real-world scenes of inland waterways. Examples of the images and corresponding disparity maps generated by SGM are shown in Fig. 10. It should be emphasized that the disparity map of the water surface areas cannot be accurately estimated by common algorithms because the disparity map of water surface areas is estimated based on the keypoints extracted from the reflection of the bankside objects. At present, we evaluate the stereo matching algorithm mainly on the nonwater areas, as no ground-truth data for the water area are provided and we think that to enable collision avoidance, the depth information of the bankside area is more important.

\section{Water Segmentation}

For water segmentation, we apply Deeplab v3+ [54], which is an effective model for semantic segmentation tasks. We evaluate the model on the high- and low-resolution images separately in our water segmentation benchmark. We also evaluate the model independently on the images of both resolutions. The training set and test set contain images captured in different scenes and under different weather conditions. For evaluation, we refer to the evaluation metrics of the lane detection task [55]. The number of images in the training set and test set as well as the test results are shown in Table. III

We present images with low-accuracy results in Fig. 11 to illustrate the difficulties in the water segmentation task. The high degree of variability of different inland water environments makes it difficult to build a robust model for water segmentation [56]. Also, unlike lane detection tasks for autonomous vehicles, the similarity between the appearance of objects on the banks and the reflection makes it difficult to accurately segment the water area. 
TABLE II: Result of stereo matching based on SGM

\begin{tabular}{c|c|c|c|c|c}
\hline Threshold & $5 \mathrm{px}$ & $4 \mathrm{px}$ & $3 \mathrm{px}$ & $2 \mathrm{px}$ & $1 \mathrm{px}$ \\
\hline Out-All & 6.880 & 9.286 & 12.565 & 19.142 & 48.803 \\
\hline
\end{tabular}

Out-All: Percentage of erroneous pixels in total

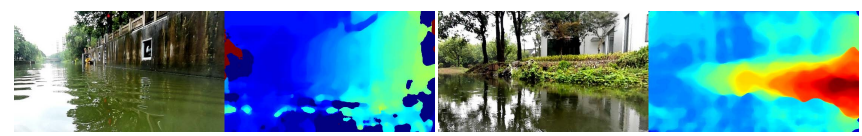

Fig. 10: Disparity map of relatively unacceptable (left) and good (right) results for stereo matching based on SGM.

\section{CONCLUSION}

We present the USVInland dataset, the first multisensor USV dataset for inland waterways. The dataset is collected under various weather conditions to represent the real-world driving scenes in inland waterways. Based on our dataset, we build benchmarks for SLAM, stereo matching and water segmentation tasks. After evaluating typical algorithms on our dataset, we find that common approaches for autonomous driving may not perform well when applied to inland waterway scenes and do not meet the requirements for the safe navigation of USVs. By publishing the USVInland dataset, we hope that this work will support researchers interested in USVs for inland waterways and spur advancement in this field. In the future, we plan to further extend our dataset. For example, historical data will also be included to enable research on the long-term SLAM of USVs in real-world inland waterways.

\section{REFERENCES}

[1] J. Han, Y. Cho, and J. Kim, "Coastal slam with marine radar for usv operation in gps-restricted situations," IEEE Journal of Oceanic Engineering, vol. 44, no. 2, pp. 300-309, 2019.

[2] Y. Peng, Y. Yang, J. Cui, X. Li, H. Pu, J. Gu, S. Xie, and J. Luo, "Development of the usv 'jinghai-i'and sea trials in the southern yellow sea," Ocean engineering, vol. 131, pp. 186-196, 2017.

[3] T. Pastore and V. Djapic, "Improving autonomy and control of autonomous surface vehicles in port protection and mine countermeasure scenarios," Journal of Field Robotics, vol. 27, no. 6, pp. 903-914, 2010.

[4] D. Madeo, A. Pozzebon, C. Mocenni, and D. Bertoni, "A low-cost unmanned surface vehicle for pervasive water quality monitoring," IEEE Transactions on Instrumentation and Measurement, vol. 69 , no. 4, pp. 1433-1444, 2020.

[5] N. Ruangpayoongsak, J. Sumroengrit, and M. Leanglum, "A floating waste scooper robot on water surface," in 2017 17th International Conference on Control, Automation and Systems (ICCAS), pp. 15431548, IEEE, 2017.

[6] W. Wang, B. Gheneti, L. A. Mateos, F. Duarte, C. Ratti, and D. Rus, "Roboat: An autonomous surface vehicle for urban waterways," in 2019 IEEE/RSJ International Conference on Intelligent Robots and Systems (IROS), pp. 6340-6347, IEEE, 2019.

[7] T. Kriechbaumer, K. Blackburn, T. P. Breckon, O. Hamilton, and M. Rivas Casado, "Quantitative evaluation of stereo visual odometry for autonomous vessel localisation in inland waterway sensing applications," Sensors, vol. 15, no. 12, pp. 31869-31887, 2015.

[8] J. Fuentes-Pacheco, J. Ruiz-Ascencio, and J. M. Rendón-Mancha, "Visual simultaneous localization and mapping: a survey," Artificial intelligence review, vol. 43, no. 1, pp. 55-81, 2015.

[9] M. Bijelic, T. Gruber, and W. Ritter, "A benchmark for lidar sensors in fog: Is detection breaking down?," in 2018 IEEE Intelligent Vehicles Symposium (IV), pp. 760-767, IEEE, 2018.
TABLE III: Result of water segmentation on DeepLab v3+

\begin{tabular}{c||c|c|c}
\hline Resolution & Low-Res & High-Res & Both \\
\hline Training Num & 345 & 121 & 466 \\
Test Num & 173 & 61 & 234 \\
MaxF[\%] & 94.52 & 96.02 & 95.80 \\
AvgPrec[\%] & 94.12 & 95.13 & 94.99 \\
PRE[\%] & 99.11 & 99.55 & 98.93 \\
REC[\%] & 90.33 & 92.32 & 93.28 \\
FPR[\%] & 0.65 & 0.43 & 1.27 \\
FNR[\%] & 9.67 & 7.68 & 6.72 \\
\hline
\end{tabular}

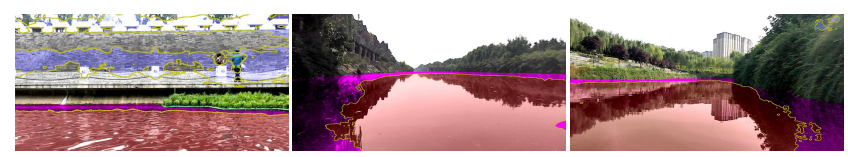

Fig. 11: Examples of the water segmentation results based on DeepLab v3+: Red area: true positive. Purple area: false negative. Blue area: false positive. The yellow line shows the estimated waterline. The image on the left shows that, for one image in the test set, the wall on the bank is falsely detected as water. The other two images show that it is difficult to accurately segment the water area especially for areas near the water boundaries.

[10] W. Wang, T. Shan, P. Leoni, D. Fernandez-Gutierrez, D. Meyers, C. Ratti, and D. Rus, "Roboat ii: A novel autonomous surface vessel for urban environments," arXiv preprint arXiv:2007.10220 2020.

[11] A. Geiger, P. Lenz, and R. Urtasun, "Are we ready for autonomous driving? the kitti vision benchmark suite," in 2012 IEEE Conference on Computer Vision and Pattern Recognition, pp. 3354-3361, IEEE, 2012

[12] W. Maddern, G. Pascoe, C. Linegar, and P. Newman, "1 year, 1000 km: The oxford robotcar dataset," The International Journal of Robotics Research, vol. 36, no. 1, pp. 3-15, 2017.

[13] M. Cordts, M. Omran, S. Ramos, T. Rehfeld, M. Enzweiler, R. Benenson, U. Franke, S. Roth, and B. Schiele, "The cityscapes dataset for semantic urban scene understanding," in Proceedings of the IEEE conference on computer vision and pattern recognition, pp. 3213$3223,2016$.

[14] A. Robicquet, A. Sadeghian, A. Alahi, and S. Savarese, "Learning social etiquette: Human trajectory understanding in crowded scenes," in European conference on computer vision, pp. 549-565, Springer, 2016.

[15] M. Barekatain, M. Martí, H.-F. Shih, S. Murray, K. Nakayama, Y. Matsuo, and H. Prendinger, "Okutama-action: An aerial view video dataset for concurrent human action detection," in Proceedings of the IEEE Conference on Computer Vision and Pattern Recognition Workshops, pp. 28-35, 2017.

[16] M. M. Zhang, J. Choi, K. Daniilidis, M. T. Wolf, and C. Kanan, "Vais: A dataset for recognizing maritime imagery in the visible and infrared spectrums," in Proceedings of the IEEE Conference on Computer Vision and Pattern Recognition Workshops, pp. 10-16, 2015.

[17] D. K. Prasad, D. Rajan, L. Rachmawati, E. Rajabally, and C. Quek, "Video processing from electro-optical sensors for object detection and tracking in a maritime environment: a survey," IEEE Transaction on Intelligent Transportation Systems, vol. 18, no. 8, pp. 1993-2016, 2017.

[18] J. Taipalmaa, N. Passalis, H. Zhang, M. Gabbouj, and J. Raitoharju, "High-resolution water segmentation for autonomous unmanned surface vehicles: a novel dataset and evaluation," in 2019 IEEE 29th International Workshop on Machine Learning for Signal Processing $(M L S P)$, pp. 1-6, IEEE, 2019.

[19] B. Bovcon, J. Muhovič, J. Perš, and M. Kristan, "The mastr1325 dataset for training deep usv obstacle detection models," in 2019 IEEE/RSJ International Conference on Intelligent Robots and Systems (IROS), pp. 3431-3438, IEEE, 2019

[20] A. Shojaei, H. I. Moud, and I. Flood, "Proof of concept for the use of 
small unmanned surface vehicle in built environment management," in Proceeding of Construction Research Congress, pp. 148-157, 2018.

[21] M. Dunbabin, A. Grinham, and J. Udy, "An autonomous surface vehicle for water quality monitoring," in Australasian conference on robotics and automation (ACRA), pp. 2-4, Citeseer, 2009.

[22] A. Akib, F. Tasnim, D. Biswas, M. B. Hashem, K. Rahman, A. Bhattacharjee, and S. A. Fattah, "Unmanned floating waste collecting robot," in TENCON 2019-2019 IEEE Region 10 Conference (TENCON), pp. 2645-2650, IEEE, 2019.

[23] J. Zhang and S. Singh, "Loam: Lidar odometry and mapping in realtime.," in Robotics: Science and Systems, no. 9, 2014.

[24] W. Hess, D. Kohler, H. Rapp, and D. Andor, "Real-time loop closure in 2d lidar slam," in 2016 IEEE International Conference on Robotics and Automation (ICRA), pp. 1271-1278, IEEE, 2016.

[25] T. Shan and B. Englot, "Lego-loam: Lightweight and groundoptimized lidar odometry and mapping on variable terrain," in 2018 IEEE/RSJ International Conference on Intelligent Robots and Systems (IROS), pp. 4758-4765, IEEE, 2018

[26] R. Mur-Artal, J. M. M. Montiel, and J. D. Tardos, "Orb-slam: a versatile and accurate monocular slam system," IEEE transactions on robotics, vol. 31, no. 5, pp. 1147-1163, 2015.

[27] T. Qin, P. Li, and S. Shen, "Vins-mono: A robust and versatile monocular visual-inertial state estimator," IEEE Transactions on Robotics, vol. 34, no. 4, pp. 1004-1020, 2018.

[28] M. Meyer and G. Kuschk, "Automotive radar dataset for deep learning based 3d object detection," in 2019 16th European Radar Conference (EuRAD), pp. 129-132, IEEE, 2019.

[29] D. Barnes, M. Gadd, P. Murcutt, P. Newman, and I. Posner, "The oxford radar robotcar dataset: A radar extension to the oxford robotcar dataset," arXiv preprint arXiv:1909.01300 2019.

[30] D. Vivet, P. Checchin, and R. Chapuis, "Localization and mapping using only a rotating fmcw radar sensor," Sensors, vol. 13 , no. 4 , pp. 4527-4552, 2013.

[31] F. Schuster, C. G. Keller, M. Rapp, M. Haueis, and C. Curio, "Landmark based radar slam using graph optimization," in 2016 IEEE 19th International Conference on Intelligent Transportation Systems (ITSC), pp. 2559-2564, IEEE, 2016.

[32] J. Han and J. Kim, "Navigation of an unmanned surface vessel under bridges," in 2013 10th International Conference on Ubiquitous Robots and Ambient Intelligence (URAI), pp. 206-210, IEEE, 2013.

[33] T. Shan, B. Englot, D. Meyers, W. Wang, C. Ratti, and D. Rus, "Lio-sam: Tightly-coupled lidar inertial odometry via smoothing and mapping," arXiv preprint arXiv:2007.00258 2020.

[34] L. Hong and G. Chen, "Segment-based stereo matching using graph cuts," in Proceedings of the 2004 IEEE Computer Society Conference on Computer Vision and Pattern Recognition, 2004. CVPR 2004., vol. 1, pp. I-I, IEEE, 2004

[35] H. Hirschmuller, "Stereo processing by semiglobal matching and mutual information," IEEE Transactions on pattern analysis and machine intelligence, vol. 30, no. 2, pp. 328-341, 2007.

[36] J. Žbontar and Y. LeCun, "Stereo matching by training a convolutional neural network to compare image patches," The journal of machine learning research, vol. 17, no. 1, pp. 2287-2318, 2016.

[37] W. Luo, A. G. Schwing, and R. Urtasun, "Efficient deep learning for stereo matching," in Proceedings of the IEEE Conference on Computer Vision and Pattern Recognition, pp. 5695-5703, 2016.

[38] G. Yang, H. Zhao, J. Shi, Z. Deng, and J. Jia, "Segstereo: Exploiting semantic information for disparity estimation," in Proceedings of the European Conference on Computer Vision (ECCV), pp. 636-651, 2018.

[39] H. Wang and Z. Wei, "Stereovision based obstacle detection system for unmanned surface vehicle," in 2013 IEEE International Conference on Robotics and Biomimetics (ROBIO), pp. 917-921, IEEE, 2013.

[40] B. Bovcon and M. Kristan, "Obstacle detection for usvs by joint stereo-view semantic segmentation," in 2018 IEEE/RSJ International Conference on Intelligent Robots and Systems (IROS), pp. 5807-5812, IEEE, 2018.

[41] W. Zhan, C. Xiao, H. Yuan, and Y. Wen, "Effective waterline detection for unmanned surface vehicles in inland water," in 2017 Seventh International Conference on Image Processing Theory, Tools and Applications (IPTA), pp. 1-6, IEEE, 2017.

[42] X. Zou, C. Xiao, W. Zhan, C. Zhou, S. Xiu, and H. Yuan, "A novel water-shore-line detection method for usv autonomous navigation," Sensors, vol. 20, no. 6, p. 1682, 2020.
[43] W. Zhan, C. Xiao, Y. Wen, C. Zhou, H. Yuan, S. Xiu, Y. Zhang, X. Zou, X. Liu, and Q. Li, "Autonomous visual perception for unmanned surface vehicle navigation in an unknown environment," Sensors, vol. 19, no. 10, p. 2216, 2019.

[44] B. Bovcon and M. Kristan, "A water-obstacle separation and refinement network for unmanned surface vehicles," arXiv preprint arXiv:2001.01921 2020.

[45] UBlox, ZED-F9P Moving Base Applications: Application Note, 2020. https://www.u-blox.com/sites/default/files/ZED-F9PMovingBase_AppNote_\%28UBX19009093\%29.pdf

[46] P. Sun, H. Kretzschmar, X. Dotiwalla, A. Chouard, V. Patnaik, P. Tsui, J. Guo, Y. Zhou, Y. Chai, B. Caine, et al., "Scalability in perception for autonomous driving: Waymo open dataset," in Proceedings of the IEEE/CVF Conference on Computer Vision and Pattern Recognition, pp. 2446-2454, 2020.

[47] Aaron20127, "Camera Lidar Joint Calibration." https://github.com/Aaron20127/Camera-lidar-joint-calibration 2019.

[48] D. Chetverikov, D. Svirko, D. Stepanov, and P. Krsek, "The trimmed iterative closest point algorithm," in Object recognition supported by user interaction for service robots, vol. 3, pp. 545-548, IEEE, 2002.

[49] K. Wada, "labelme: Image Polygonal Annotation with Python," 2016. https://github.com/wkentaro/labelme

[50] R. Mur-Artal and J. D. Tardós, "Orb-slam2: An open-source slam system for monocular, stereo, and rgb-d cameras," IEEE Transactions on Robotics, vol. 33, no. 5, pp. 1255-1262, 2017.

[51] R. Mur-Artal, J. D. Tardos, J. M. M. Montiel, and D. G.-L. (DBoW2), "ORB-SLAM2." https://github.com/raulmur/ORB_SLAM2 2016.

[52] A. Chambers, S. Achar, S. Nuske, J. Rehder, B. Kitt, L. Chamberlain, J. Haines, S. Scherer, and S. Singh, "Perception for a river mapping robot," in 2011 IEEE/RSJ International Conference on Intelligent Robots and Systems, pp. 227-234, IEEE, 2011.

[53] F. Schuster, M. Wörner, C. G. Keller, M. Haueis, and C. Curio, "Robust localization based on radar signal clustering," in 2016 IEEE Intelligent Vehicles Symposium (IV), pp. 839-844, IEEE, 2016.

[54] L.-C. Chen, Y. Zhu, G. Papandreou, F. Schroff, and H. Adam, "Encoder-decoder with atrous separable convolution for semantic image segmentation," in Proceedings of the European conference on computer vision (ECCV), pp. 801-818, 2018.

[55] J. Fritsch, T. Kuehnl, and A. Geiger, "A new performance measure and evaluation benchmark for road detection algorithms," in 16th International IEEE Conference on Intelligent Transportation Systems (ITSC 2013), pp. 1693-1700, IEEE, 2013.

[56] S. Scherer, J. Rehder, S. Achar, H. Cover, A. Chambers, S. Nuske, and S. Singh, "River mapping from a flying robot: state estimation, river detection, and obstacle mapping," Autonomous Robots, vol. 33, no. 1-2, pp. 189-214, 2012. 